\title{
Increased neutrophil-to-lymphocyte ratio is associated with disease-specific mortality in patients with penile cancer
}

\author{
Jun Kasuga', Takashi Kawahara ${ }^{1,2^{*}}$ (D, Daiji Takamoto ${ }^{3}$, Sachi Fukui ${ }^{4}$, Takashi Tokita $^{5}$, Tomoyuki Tadenuma ${ }^{6}$, \\ Masaki Narahara ${ }^{7}$, Syusei Fusayasu ${ }^{8}$, Hideyuki Terao ${ }^{9}$, Koji Izumi $^{10}$, Hiroki Ito ${ }^{1}$, Yusuke Hattori ${ }^{2}$, Jun-ichi Teranishi ${ }^{2}$, \\ Takeshi Sasaki ${ }^{11}$, Kazuhide Makiyama', Yasuhide Miyoshi ${ }^{2}$, Masahiro Yao ${ }^{1}$, Yasushi Yumura², \\ Hiroshi Miyamoto $^{12}$ and Hiroji Uemura ${ }^{2}$
}

\begin{abstract}
Background: The neutrophil-to-lymphocyte ratio (NLR), a simple marker of the systemic inflammatory response, has been demonstrated to correlate with patient outcomes for various solid malignancies. We investigated the utility of the pretreatment NLR as a prognosticator in patients who presented with penile cancer.

Methods: A total of 41 patients who underwent complete blood count with differential and subsequent radical penectomy from 1988 to 2014 were analyzed. We assessed the correlation between the NLR and the prognosis of penile cancer.

Results: The median and mean ( \pm SD) NLRs in 41 penile cancer patients were 3.42 and $5.03 \pm 4.99$, respectively. Based on the area under receiver operator characteristic curve, the cut-off value of NLR was determined to be 2.82 . Patients with a high NLR ( 22.82$)$ showed a significantly poorer cancer-specific survival $(p=0.023)$ than those with a low NLR.

Conclusions: The pretreatment NLR may function as a biomarker that precisely predicts the prognosis in patients with penile cancer.
\end{abstract}

Keywords: Penile cancer, Biomarker, Neutrophil-to-lymphocyte ratio, Immunohistochemistry

\section{Background}

Penile squamous cell carcinoma (PSCC) is a rare disease in developed countries, with an incidence of $0.3-1.0$ per 100,000 males in Europe and North America and 0.40.5 per 100,000 males in Japan $[1,2]$. However, it represents an important public health problem for developing countries in Asia, Africa and South America, where its incidence varies from 3 to 8.3 cases per 100,000 [3]. The major prognostic factors in PSCC are tumor grade and the presence of perineal and lymphatic invasion [4]. SCC, a soluble epithelial antigen and a classical molecular

\footnotetext{
*Correspondence: takashi_tk2001@yahoo.co.jp

${ }^{1}$ Department of Urology, Yokohama City University Graduate School of Medicine, 3-9 Fukuura, Kanazawa-ku, Yokohama, Kanagawa 2360004, Japan ${ }^{2}$ Departments of Urology and Renal transplantation, Yokohama City University Medical Center, Yokohama, Japan

Full list of author information is available at the end of the article
}

marker lacks sensitivity in the detection of small tumor burdens and has little prognostic significance in survival after surgery [5]. The overexpression of p53 and Ki-67 and the loss of membranous E-cadherin determined immunohistochemically in biopsy or penectomy tissue specimens are also shown to associate with the detection of lymph node metastases, but these markers are not useful in clinical practice $[4,6]$.

The neutrophil-to-lymphocyte ratio (NLR) has been suggested as a simple marker of the systemic inflammatory response in critical care patients [7]. It has also been reported as an independent prognostic factor for several solid malignancies [8-17]. Importantly, the NLR can easily be calculated from routine complete blood counts $(\mathrm{CBCs})$ in peripheral blood samples $[15,16]$. 
We investigated the utility of the pretreatment NLR as a prognosticator in patients who presented with penile cancer.

\section{Methods}

\section{Patients}

A total of 73,637 CBC exams, which included absolute neutrophil and lymphocyte counts, were performed in 9782 male patients at the Department of Urology, Yokohama City University Hospital (Yokohama, Japan) from 1999 to 2015. Among these, we investigated the NLRs in patients with urological diseases, such as penile cancer, renal cell carcinoma, prostate cancer, testicular cancer, overactive bladder, and benign prostatic hyperplasia (Fig. 1).

We conducted a retrospective study of 41 patients with penile cancer who underwent $\mathrm{CBCs}$ and subsequent radical penectomy at Yokohama City University Medical Center and 24 other institutions from 1988 to 2014. Some of the patients who were diagnosed at Yokohama City University Hospital overlapped with those in a study of 9782 male patients. All of the patients were pathologically diagnosed with PSCC. Patients were followed-up for 2.3271.7 months (median: 34.7 months). The incidence of tumor relapse and patient outcomes during the follow-up period were evaluated. This study was approved by the ethics committee of Yokohama City University, Yokohama City University Medical Center and the other participating institutions approved the protocol of the present study. Written informed consent was obtained from the penile cancer patients.

\section{Clinical and laboratory assessments}

The NLR was calculated using the neutrophil and lymphocyte counts, which were obtained via CBCs before the surgery. We determined the cut-off point of the NLR according to the sensitivity and specificity levels derived from area under receiver operator characteristic (AUROC) curve.

\section{Immunohistochemistry}

An immunohistochemical analysis was performed in 5$\mu \mathrm{m}$-thick tissue microarray (TMA) sections, including 183 penile tissue specimens that were obtained from US Biomax (PE2081, Rockville, MD). Immunohistochemical staining was performed., as described previously [18], using a primary antibody to CD66b (clone G10F5, diluted to 1:200, BD Biosciences, San Jose, CA, USA) or CD8 (clone $\mathrm{C} 8 / 144 \mathrm{~B}$, diluted to $1: 100$, DAKO Corporation, Carpenteria, CA, USA), to detect tumor-infiltrating neutrophils and lymphocytes, respectively. The slides were then examined by a single pathologist (HM) who was blinded to the identity of the samples. The total numbers of CD66b-positive and CD8-positive cells were counted in each TMA core. Due to the small number benign specimens and some of the specimens including infection, we did not compare between benign and malignant specimens.

\section{Statistical analyses}

The patients' characteristics and preoperative factors were analyzed using the Mann-Whitney $U$ and chi-squared tests, using the Graph Pad Prism software program (Graph Pad Software, La Jolla, CA, USA). The survival duration was defined as the time between the dates of pathological diagnosis and tumor progression or death. A log-rank test was performed for comparison between higher and lower NLR groups. Multivariate logistic regression models were used to detect the individual factors. $P$ values of $<0.05$ were considered to indicate statistical significance.

\section{Results}

Penile cancer patients showed a higher NLR

According to the clinical database of the Department of Urology at Yokohama City University Hospital, 9782 patients underwent an NLR check during the study period. The median/mean NLRs for each disease (mainly the names of the diagnosed diseases which were used for

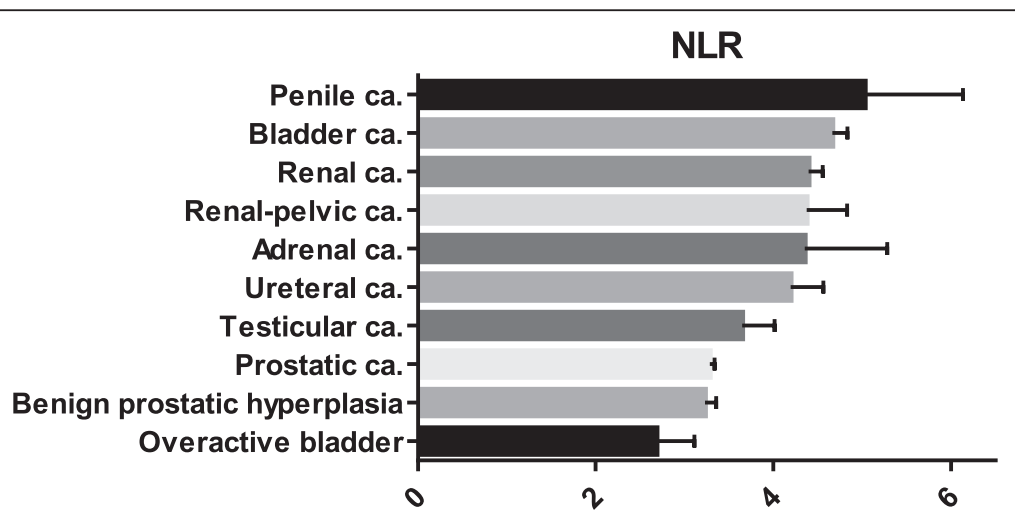

Fig. 1 The NLRs in patients with urological diseases. Each value represents the mean and $95 \%$ confidence interval 
medical insurance) were 3.42/5.03 (penile cancer), 2.67/ 4.67 (bladder cancer), 2.64/4.40 (renal cell carcinoma), (2.39/4.37 (renal pelvic cancer), 3.58/4.35 (adrenal cancer), 2.51/4.20 (ureteral cancer), 2.39/3.65 (testicular cancer), 2.26/3.28 (prostatic cancer), 2.22/3.23 (benign prostatic hyperplasia), and 2.41/2.68 (overactive bladder) (Fig. 1). The NLR of the PSCC patients was significantly higher than the NLRs of testicular cancer $(p<0.05)$, prostate cancer $(p<0.01)$, benign prostate hyperplasia $(p<0.01)$, and overactive bladder $(p<0.001)$ patients.

\section{The NLR predicts the survival of penile cancer patients}

The median and mean $( \pm$ SD) ages of the 41 patients were 69 and $68.5( \pm 11.4)$ years with median and mean ( \pm SD) follow-up periods of 34.7 and $60.7 \quad( \pm 52.1)$ months after the initial diagnosis. The clinicopathological data of these patients are summarized in Table 1 .

Table 1 Patients' characteristics

\begin{tabular}{|c|c|c|c|c|}
\hline \multirow[t]{2}{*}{ Variables } & \multicolumn{3}{|c|}{ number or median (mean \pm SD) } & \multirow[t]{2}{*}{$p$ value } \\
\hline & all & $\mathrm{NLR}<2.82$ & $N L R \geq 2.82$ & \\
\hline $\begin{array}{l}\text { A number of } \\
\text { patients. }\end{array}$ & $41(100.0 \%)$ & $21(51.2 \%)$ & $20(48.8 \%)$ & \\
\hline Age (years) & $69(68.5 \pm 11.8)$ & $69(66.3 \pm 10.9)$ & $71(70.7 \pm 12.5)$ & 0.243 \\
\hline \multicolumn{5}{|l|}{ Location } \\
\hline Gland & $30(73.2 \%)$ & $15(57.1 \%)$ & $15(75.0 \%)$ & \multirow[t]{4}{*}{0.287} \\
\hline Foreskin & $8(19.5 \%)$ & $5(23.8 \%)$ & $3(15.0 \%)$ & \\
\hline Shaft & $2(4.9 \%)$ & $0(0.0 \%)$ & $2(10.0 \%)$ & \\
\hline Unknown & $1(2.4 \%)$ & $1(4.8 \%)$ & $0(0.0 \%)$ & \\
\hline \multicolumn{5}{|c|}{ Tumor grade (differentiation) } \\
\hline Well & $26(63.4 \%)$ & $15(57.1 \%)$ & $11(55.0 \%)$ & \multirow[t]{4}{*}{0.330} \\
\hline Moderate & $10(24.0 \%)$ & $3(14.3 \%)$ & $7(35.0 \%)$ & \\
\hline Poor & $2(4.9 \%)$ & $1(4.8 \%)$ & $1(5.0 \%)$ & \\
\hline Unknown & $3(7.3 \%)$ & $2(9.5 \%)$ & $1(5.0 \%)$ & \\
\hline \multicolumn{5}{|c|}{ Pathological T stage } \\
\hline 1 & $22(53.7 \%)$ & $14(66.7 \%)$ & $8(40.0 \%)$ & \multirow[t]{5}{*}{0.273} \\
\hline 2 & $13(31 . \%)$ & $4(19.0 \%)$ & $9(45.0 \%)$ & \\
\hline 3 & $2(4.9 \%)$ & $1(4.8 \%)$ & $1(5.0 \%)$ & \\
\hline 4 & $3(7.3 \%)$ & $1(4.8 \%)$ & $2(10.0 \%)$ & \\
\hline Unknown & $1(2.4 \%)$ & 1 (4.8 \%) & $0(0.0 \%)$ & \\
\hline $\begin{array}{l}\text { Lymph node } \\
\text { metastasis }\end{array}$ & 9 (21.6 \%) & $2(9.5 \%)$ & 7 (35.0 \%) & 0.049 \\
\hline $\begin{array}{l}\text { Distant } \\
\text { metastasis }\end{array}$ & $1(2.4 \%)$ & 1 (4.8\%) & $0(0.0 \%)$ & 0.323 \\
\hline \multicolumn{5}{|c|}{ Anatomic stage } \\
\hline । & 17 (41.5 \%) & $10(47.6 \%)$ & 7 (35.0 \%) & \multirow[t]{5}{*}{0.214} \\
\hline$\|$ & 14 (34.1\%) & $8(38.1 \%)$ & $6(30.0 \%)$ & \\
\hline III & $3(7.3 \%)$ & $0(0.0 \%)$ & $3(15.0 \%)$ & \\
\hline IV & $6(14.6 \%)$ & $2(9.5 \%)$ & $4(20.0 \%)$ & \\
\hline Unknown & $1(2.4 \%)$ & $1(4.8 \%)$ & $0(0.0 \%)$ & \\
\hline
\end{tabular}

We created an AUROC curve to determine the NLR cut-off value for predicting the prognosis of penile cancer patients. The cut-off value was determined to be 2.82. High NLR was significantly associated with lymph node metastasis $(p=0.049)$. Patients with high NLRs also showed a significantly poorer cancer-specific survival $(p=0.023)$ than those with low NLRs (Fig. 2a). In addition, patients with high NLRs tended to correlate with poorer overall survival $(p=0.076)$ (Fig. $2 \mathrm{~b})$. We performed a multivariate analysis but it did not reveal any significant independent factors that predicted the prognosis.

Tumor-infiltrating neutrophils and lymphocytes in PSCC Immunohistochemistry was used to determine the numbers of tumor-infiltrating neutrophils and lymphocytes in the penile TMA. Both CD66b- and CD8-positive immune cells were present in the specimens (Fig. 3). We then analyzed the relationship between the number of tumorinfiltrating CD66b-positive neutrophils or CD8-positive lymphocytes and tumor grade or stage. There were no statistically significant correlations between the number of CD66b- or CD8-positive cells and tumor grade. Interestingly, the number of CD66b-positive cells, but not that of CD8-positive cells, was significantly lower in high stage disease, compared with stage 1 disease $(p=0.014)$. However, there were no statistically significant differences in the ratio of CD66b/CD8-positive cell Table 2.

\section{Discussion}

We have evaluated the pretreatment NLR as a predictor of survival in penile cancer patients. We found that high NLRs were associated with a poorer prognosis of penile cancer.

Several prognostic factors have been established for patients with penile cancer. Nodal metastasis is the most important predictor of a poor clinical outcome [4]. Tumor grade and perineural or lymphatic invasion are also known prognostic predictors. p53, Ki-67, E-cadherin, and epidermal growth factor receptor (EGFR) are considered to be molecular prognostic markers, but they are not always useful in clinical practice $[4,6]$.

It has been suggested that the NLR can be used to estimate the magnitude of systemic inflammation in cancer patients $[8,18-20]$. The NLR is easily and inexpensively measured [21]. An elevated NLR has been reported to be associated with a poorer survival rate in a variety of cancers $[8-13,22]$.

Pond et al. demonstrated that the NLR was significantly associated with survival in 26 patients with penile cancer [21]. This has been the only paper to describe the relationship between the NLR and the prognosis of penile cancer; however, the subjects were limited to patients who were undergoing concurrent chemo-radiotherapy. 

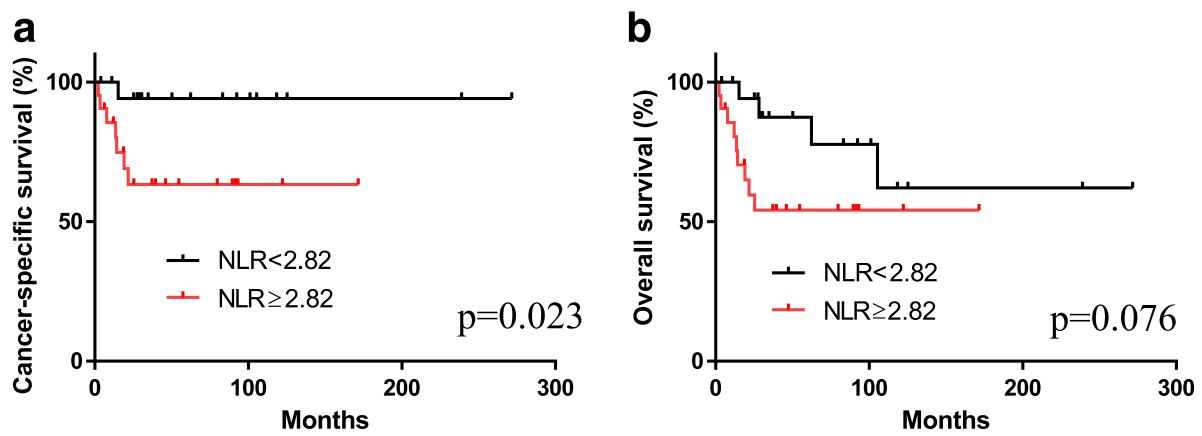

Fig. 2 The correlation between the NLR and $\mathbf{a}$ cancer-specific survival or $\mathbf{b}$ overall survival

The AUROC determined the cut-off value of the NLR to be 2.82 in the present study. Several studies in patients with advanced pancreatic cancer have shown NLR cut-off values of approximately five [8]. In patients with intrahepatic cholangiocarcinoma and those with liver metastasis from colorectal carcinoma [9], the NLR cutoff value is also set at five. In urological cancers, an NLR cut-off value of approximately five has been used for prostate cancer, while scores of 2 to 5 have been used for renal cell carcinoma [23]. Our cut-off point for the NLR was thus somewhat lower than the values determined in previous studies, despite the fact that the NLR for penile cancer was high in comparison to other urological diseases. Of note, in most of these studies [ref], the NLR was assessed in advanced cases. On the other hand, in our prevous study [24], the NLR cut-off point for predicting the prognosis of patients most of who had organ-confined prostate cancer was 2.4 .

In recent studies, the preoperative levels of $\mathrm{C}$-reactive protein (CRP) were found to predict survival in patients with penile squamous cell carcinoma $[24,25]$. In various tumors, other markers of the systemic inflammatory response have also been developed to predict patient outcomes, such as the platelet-to-lympocyte ratio (PLR), the lympocyte-to-monocyte ratio (LMR), and the preoperative haemogolobin and albumin levels [26-31]. It is necessary to investigate the relationship between these markers and the prognosis of penile cancer in the future.

The management of the regional lymph nodes in penile cancer patients is highly important for long-term survival. However, there is no non-invasive or minimally invasive staging technique that can be used to determine their lymph node status. Proven molecular markers or accurate minimally invasive tests which can be used to identify nodal metastasis are desired.

The present study is associated with some limitations due to its retrospective nature. Our patients received a variety of therapies, including surgery, chemotherapy, radiation therapy, other treatments, and their combinations. Although the treatment options were heterogeneous, we found that the NLR was associated with patient outcomes. Second is that we did not perform mechanistic experiments to determine the roles of neutrophils and/or lymphocytes in penile cancer progression. Nonetheless, the current results support the findings of previous studies indicating correlations between the NLR/inflammation and the clinical outcome of patients with several types of advanced-stage solid tumors. Third, the sample size was low because of the low incidence of

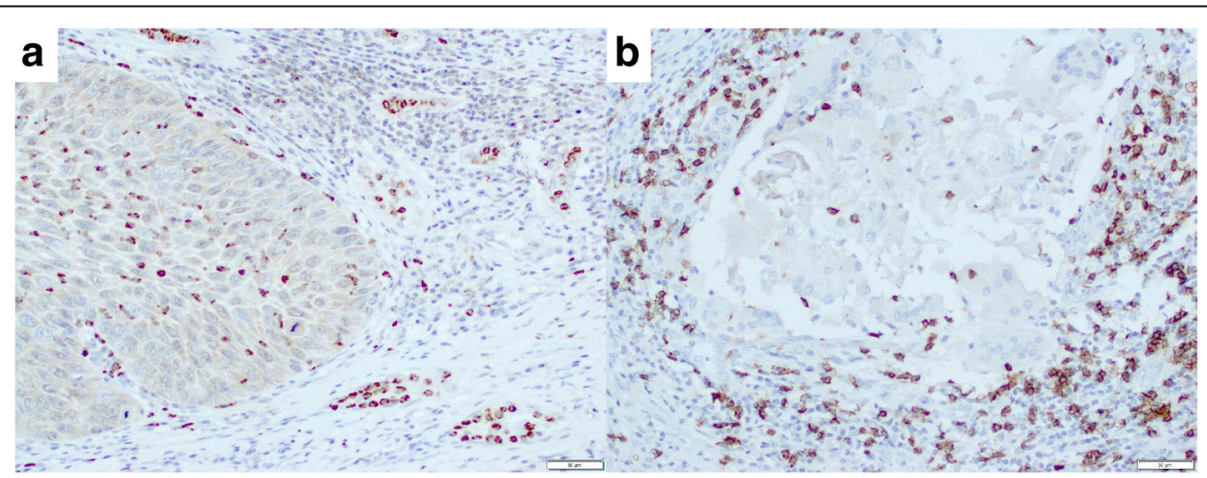

Fig. 3 Immunohistochemistry of a CD66b and $\mathbf{b}$ CD8 in penile cancer specimens. CD66b- and CD8-positive immune cells are seen predominantly in the stromal tissue 
Table 2 Tumor-infiltrating CD66b/CD8-positive immune cells in the penile TMA (PE2081)

\begin{tabular}{lllll}
\hline & \multicolumn{2}{c}{ Pathological Grade } & p value \\
\cline { 2 - 4 } & Grade 1 $(n=128)$ & Grade $2 \& 3(n=47)$ & Unknown $(n=8)$ & \\
\hline CD66b & $15(23.8 \pm 25.5)$ & $12(31.8 \pm 38.0)$ & $7.5(14.4 \pm 14.1)$ & 0.209 \\
CD8 & $62(58.3 \pm 37.1)$ & $60.5(56.8 \pm 37.5)$ & $68.5(59.9 \pm 32.0)$ & 0.898 \\
Anatomic Stage & & & \\
& Stage I $(n=147)$ & Stage II \& III $(n=36)$ & 0.014 \\
CD66b & $16(28.7 \pm 31.2)$ & $7(15.9 \pm 19.9)$ & & 0.183 \\
\hline CD8 & $65(60.0 \pm 36.5)$ & $35(49.1 \pm 38.2)$ & &
\end{tabular}

Data represent the median (mean \pm SD)

penile cancer and the lack of some information, including the degree of extranodal extension [32]. Despite this limitation, the population of the present study represents the largest number of penile cancer patients in whom the NLR was investigated. An additional limitation is that the data in the clinical database study was extracted electronically. Thus, the detailed information about the specific diseases was not confirmed, while the technique allowed us to obtain a large number of cases.

Our immunohistochemistry revealed no significant correlations between the number of tumor-infiltrating CD66b- or CD8-positive immune cells and tumor grade or stage. Nonetheless, higher number of CD66b-positive neutrophils was correlated with lower tumor stage. Wang et al. showed that increased tumor-infiltrating neutrophils and neutrophil-to-lymphocyte ratio in esophageal cancer specimens correlated with disease progression [33]. Although a large number of studies have demonstrated the prognostic value of NLR in various solid tumors, others have failed to show that of tumor-infiltrating neutrophils/lymphocytes in tissue specimens. We indeed performed immunohistochemical staining for CD66b and CD8 in bladder cancer and prostate cancer specimens, but found no significant correlations between the number of immunoreactive immune cells and patient outcomes (unpublished data).

\section{Conclusion}

NLR was found to correlate with lymph node metastasis as well as cancer-specific survival in patients with PSCC. Our data thus suggest that the NLR serves as a biomarker which predicts the patient outcomes.

\footnotetext{
Acknowledgement

We would like to thank Drs. Kazuki Kobayashi (Yokosuka Kyosai Hospital, Yokosuka, Japan), Junichi Ohta (Yokohama Municipal Citizen's Hospital, Yokohama, Japan), Kazuo Kitami (Fujisawa City Hospital, Fujisawa, Japan), Kotaro Hirai (Sagamihara National Hospital, Sagamihara, Japan), Yoshiki Hara (Odawara Municipal Hospital, Odawara, Japan), Kiyoshi Fujinami (Chigasaki Municipal Hospital, Chigasaki, Japan), Hiroshi Misaki (Yamato Municipal Hospital, Yamato, Japan), Takeshi Watanabe (Kanagawa Prefectural Ashigarakami Hospital, Matsuda, Japan), Yoshiharu Ogo (Yokohama Sakae Kyosai Hospital, Yokohama, Japan), Futoshi Tsuchiya (Yokohama Minato Red Cross Hospital, Yokohama, Japan), Ichiro Ikeda (Yokohama Minami Kyosai
}

Hospital, Yokohama, Japan), Teichiro Ueki (Hadano Red Cross Hospital, Hadano, Japan), Tetsuo Murai (International Goodwill Hospital, Yokohama, JAPAN), Hitomi Kanno (Toshiba Rinkan Hospital, Sagamihara, Japan), Hideki Ouchi (Fujisawa Shonandai Hospital, Fujisawa, Japan), Koichi Udagawa (Hiratsuka Kyosai Hospital, Hiratsuka, Japan), Kimio Chiba (Kawasaki Municipal Ida Hospital, Kawasaki, Japan), Kotaro Suzuki (Saiseikai Yokohama City Southern Hospital, Yokohama, Japan), Yoshitake Kato (Yokohama Hodogaya Central Hospital, Yokohama, Japan) and Takeshi Kishida (Kanagawa Cancer Center, Yokohama, Japan).

\section{Funding}

Grants from the Uehara Memorial Foundation, the Tokyo Biochemical Research Foundation, the Japanese Foundation for Research and Promotion of Endoscopy, and International exchange Grant from Kato Memorial Bioscience Foundation were provided to T.K. No grant numbers were applied.

\section{Availability of data and materials}

Due to ethical restrictions, the raw data that was used in this study is available upon request from the corresponding author.

\section{Authors' contributions}

JK, TK, YY, HM, HU conceived and designed the experiments. DT, SFuk, TTo, TTa, MN, SFus, HT, KI, YH, JT, TS, KM, YM, MY performed the experiments. JK, TK, HI, $\mathrm{HM}$ wrote the paper. All authors have read and approved the manuscript.

\section{Competing interests}

The authors declare that they have no competing interests.

\section{Consent to publish}

This study was approved by the ethics committee of Yokohama City University Medical Center including the permission to publish.

\section{Ethics approval and consent to participate}

This study was approved by the ethics committee of Yokohama City University Medical Center.

\section{Author details}

${ }^{1}$ Department of Urology, Yokohama City University Graduate School of Medicine, 3-9 Fukuura, Kanazawa-ku, Yokohama, Kanagawa 2360004, Japan. ${ }^{2}$ Departments of Urology and Renal transplantation, Yokohama City University Medical Center, Yokohama, Japan. ${ }^{3}$ Department of Urology, Yokohama Minami Kyosai Hospital, Yokohama, Japan. ${ }^{4}$ Department of Urology, Yokohama Minato Red Cross Hospital, Yokohama, Japan. ${ }^{5}$ Department of Urology, Yokosuka Kyosai Hospital, Yokosuka, Japan. ${ }^{6}$ Department of Urology, Kanagawa Cancer Center, Yokohama, Japan. ${ }^{7}$ Department of Urology, International Goodwill Hospital, Yokohama, Japan. ${ }^{8}$ Department of Urology, Yamato Municipal Hospital, Yamato, Japan. ${ }^{9}$ Department of Urology, Fujisawa City Hospital, Fujisawa, Japan.

${ }^{10}$ Department of Urology, Yokohama Municipal Citizen's Hospital, Yokohama, Japan. "1'Department of Pathology, The University of Tokyo Graduate School of Medicine, Tokyo, Japan. ${ }^{12}$ Departments of Pathology and Urology, Johns Hopkins University School of Medicine, Baltimore, USA.

Received: 5 January 2016 Accepted: 27 June 2016

Published online: 07 July 2016

\section{References}

1. Kamidono S. Cancer of the penis and its treatment. Nihon Hinyokika Gakkai Zasshi. 1992;83(1):1-15.

2. Chaux A, Netto GJ, Rodriguez IM, Barreto JE, Oertell J, Ocampos S, et al. Epidemiologic profile, sexual history, pathologic features, and human papillomavirus status of 103 patients with penile carcinoma. World J Urol. 2013;31(4):861-7.

3. Wanick FB, Teichner TC, Silva R, Magnanini MM, Azevedo LM. Squamous cell carcinoma of the penis: clinicopathologic study of 34 cases. An Bras Dermatol. 2011;86(6):1082-91.

4. Hakenberg OW, Comperat EM, Minhas S, Necchi A, Protzel C, Watkin N, et al. EAU guidelines on penile cancer: 2014 update. Eur Urol. 2015;67(1):142-50.

5. Zhu Y, Ye DW, Yao XD, Zhang SL, Dai B, Zhang HL, et al. The value of squamous cell carcinoma antigen in the prognostic evaluation, 
treatment monitoring and followup of patients with penile cancer. J Urol. 2008;180(5):2019-23.

6. Zhu Y, Zhou XY, Yao XD, Dai B, Ye DW. The prognostic significance of p53, Ki-67, epithelial cadherin and matrix metalloproteinase-9 in penile squamous cell carcinoma treated with surgery. BJU Int. 2007;100(1):204-8.

7. Zahorec R. Ratio of neutrophil to lymphocyte counts-rapid and simple parameter of systemic inflammation and stress in critically ill. Bratisl Lek Listy. 2001;102(1):5-14.

8. Xue P, Kanai M, Mori Y, Nishimura T, Uza N, Kodama Y, et al. Neutrophil-tolymphocyte ratio for predicting palliative chemotherapy outcomes in advanced pancreatic cancer patients. Cancer Med. 2014;3(2):406-15.

9. Gomez D, Morris-Stiff G, Toogood GJ, Lodge JP, Prasad KR. Impact of systemic inflammation on outcome following resection for intrahepatic cholangiocarcinoma. J Surg Oncol. 2008;97(6):513-8.

10. Chua W, Charles KA, Baracos VE, Clarke SJ. Neutrophil/lymphocyte ratio predicts chemotherapy outcomes in patients with advanced colorectal cancer. Br J Cancer. 2011;104(8):1288-95.

11. Azab B, Bhatt VR, Phookan J, Murukutla S, Kohn N, Terjanian T, et al. Usefulness of the neutrophil-to-lymphocyte ratio in predicting short- and long-term mortality in breast cancer patients. Ann Surg Oncol. 2012;19(1):217-24.

12. Dalpiaz O, Pichler M, Mannweiler S, Martin Hernandez JM, Stojakovic T, Pummer $K$, et al. Validation of the pretreatment derived neutrophillymphocyte ratio as a prognostic factor in a European cohort of patients with upper tract urothelial carcinoma. $\mathrm{Br} J$ Cancer. 2014;110(10):2531-6.

13. Jung MR, Park YK, Jeong O, Seon JW, Ryu SY, Kim DY, et al. Elevated preoperative neutrophil to lymphocyte ratio predicts poor survival following resection in late stage gastric cancer. J Surg Oncol. 2011;104(5):504-10.

14. Demirtas A, Sabur V, Akinsal EC, Demirci D, Ekmekcioglu O, Gulmez I, et al. Can neutrophil-lymphocyte ratio and lymph node density be used as prognostic factors in patients undergoing radical cystectomy? ScientificWorldJournal. 2013;2013:703579.

15. Walsh SR, Cook EJ, Goulder F, Justin TA, Keeling NJ. Neutrophil-lymphocyte ratio as a prognostic factor in colorectal cancer. J Surg Oncol. 2005;91(3):181-4

16. Ohno Y, Nakashima J, Ohori M, Hatano T, Tachibana M. Pretreatment neutrophil-to-lymphocyte ratio as an independent predictor of recurrence in patients with nonmetastatic renal cell carcinoma. J Urol. 2010;184(3):873-8.

17. Rosenberg L, Lawlor GO, Zenlea T, Goldsmith JD, Gifford A, Falchuk KR, et al. Predictors of endoscopic inflammation in patients with ulcerative colitis in clinical remission. Inflamm Bowel Dis. 2013;19(4):779-84.

18. Stotz M, Gerger A, Eisner F, Szkandera J, Loibner H, Ress AL, et al. Increased neutrophil-lymphocyte ratio is a poor prognostic factor in patients with primary operable and inoperable pancreatic cancer. Br J Cancer. 2013;109(2):416-21.

19. Smith RA, Bosonnet L, Raraty M, Sutton R, Neoptolemos JP, Campbell F, et al. Preoperative platelet-lymphocyte ratio is an independent significant prognostic marker in resected pancreatic ductal adenocarcinoma. Am J Surg. 2009;197(4):466-72.

20. Proctor MJ, Morrison DS, Talwar D, Balmer SM, O'Reilly DS, Foulis AK, et al. An inflammation-based prognostic score (mGPS) predicts cancer survival independent of tumour site: a Glasgow inflammation outcome study. Br J Cancer. 2011;104(4):726-34.

21. Pond GR, Milowsky MI, Kolinsky MP, Eigl BJ, Necchi A, Harshman LC, et al. Concurrent chemoradiotherapy for men with locally advanced penile squamous cell carcinoma. Clin Genitourin Cancer. 2014;12(6):440-6.

22. Hermanns T, Bhindi B, Wei Y, Yu J, Noon AP, Richard PO, et al. Pre-treatment neutrophil-to-lymphocyte ratio as predictor of adverse outcomes in patients undergoing radical cystectomy for urothelial carcinoma of the bladder. Br J Cancer. 2014;111(3):444-51.

23. Wei $Y$, Jiang $Y Z$, Qian WH. Prognostic role of NLR in urinary cancers: a meta-analysis. PLoS One. 2014;9(3):e92079.

24. Al Ghazal A, Steffens S, Steinestel J, Lehmann R, Schnoeller TJ, SchulteHostede A, et al. Elevated C-reactive protein values predict nodal metastasis in patients with penile cancer. BMC Urol. 2013;13:53.

25. Steffens S, Al Ghazal A, Steinestel J, Lehmann R, Wegener G, Schnoeller TJ, et al. High CRP values predict poor survival in patients with penile cancer. BMC Cancer. 2013;13:223.

26. Chechlinska M, Kowalewska M, Nowak R. Systemic inflammation as a confounding factor in cancer biomarker discovery and validation. Nat Rev Cancer. 2010;10(1):2-3.
27. Chang $Y, A n H, X u L$, Zhu Y, Yang $Y$, Lin Z, et al. Systemic inflammation score predicts postoperative prognosis of patients with clear-cell renal cell carcinoma. Br J Cancer. 2015;113(4):626-33.

28. Fox P, Hudson M, Brown C, Lord S, Gebski V, De Souza P, et al. Markers of systemic inflammation predict survival in patients with advanced renal cell cancer. Br J Cancer. 2013;109(1):147-53.

29. Krenn-Pilko S, Langsenlehner U, Thurner EM, Stojakovic T, Pichler M, Gerger A, et al. The elevated preoperative platelet-to-lymphocyte ratio predicts poor prognosis in breast cancer patients. Br J Cancer. 2014;110(10):2524-30.

30. Hutterer GC, Stoeckigt C, Stojakovic T, Jesche J, Eberhard K, Pummer K, et al. Low preoperative lymphocyte-monocyte ratio (LMR) represents a potentially poor prognostic factor in nonmetastatic clear cell renal cell carcinoma. Urol Oncol. 2014;32(7):1041-8

31. Stotz M, Szkandera J, Stojakovic T, Seidel J, Samonigg H, Kornprat P, et al. The lymphocyte to monocyte ratio in peripheral blood represents a novel prognostic marker in patients with pancreatic cancer. Clin Chem Lab Med. 2015;53(3):499-506.

32. Wang JY, Zhu Y, Tang SX, Zhang HL, Qin XJ, Zhang SL, et al. Prognostic significance of the degree of extranodal extension in patients with penile carcinoma. Asian J Androl. 2014;16(3):437-41.

33. Wang J, Jia $Y$, Wang $N$, Zhang $X$, Tan B, Zhang G, et al. The clinical significance of tumor-infiltrating neutrophils and neutrophil-to-CD8+ lymphocyte ratio in patients with resectable esophageal squamous cell carcinoma. J Transl Med. 2014;12:7.

\section{Submit your next manuscript to BioMed Central and we will help you at every step:}

- We accept pre-submission inquiries

- Our selector tool helps you to find the most relevant journal

- We provide round the clock customer support

- Convenient online submission

- Thorough peer review

- Inclusion in PubMed and all major indexing services

- Maximum visibility for your research

Submit your manuscript at www.biomedcentral.com/submit

) Biomed Central 